\title{
Interstitial HDR brachytherapy (HDR-BT) in early stage mobile tongue cancers in young patients - Gliwice experience
}

\author{
Sylwia Kellas-Ślẹczka, MD, PhD', Brygida Białas, MD, PhD', Marek Fijałkowski, MD, PhD', Krzysztof Składowski, MD, PhD², \\ Marta Szlag, MSc', Agnieszka Cholewka, MSc ${ }^{3}$ \\ 'Department of Brachytherapy, 2First Radiotherapy Clinic, 32Department of Radiotherapy and Brachytherapy Planning, \\ Maria Skłodowska-Curie Memorial Cancer Centre and Institute of Oncology, Gliwice Branch, Poland
}

\begin{abstract}
Purpose: In early stage mobile tongue cancer radical radiotherapy offers good local control and organ preservation, which is especially important in the group of young patients. In our department, for many years HDR-BT has been performed in mobile tongue cancers as a sole treatment or as a "boost" with EBRT. The aim of the study was to show our experience with HDR-BT in early stage mobile tongue cancers among young patients.

Material and methods: From 2001 to 2006 in Maria Skłodowska-Curie Memorial Cancer Centre and Institute of Oncology, Gliwice Branch, five patients under 45 years with mobile tongue cancer were treated with HDR brachytherapy (T1N0M0 - 3/5 and T2N0M0 - 2/5); 4 with HDR brachytherapy "boost" and 1 as a sole treatment. One woman was previously treated with tumour resection, but because of positive surgical margins was referred for radiotherapy. All patients had clinically negative lymph nodes, without dissection. They were treated with interstitial HDR-BT (3-8 catheters). In 4 patients treated with HDR-BT as a "boost", total doses ranged from 18 to 21 Gy given in 6-7 fractions (twice a day, 3 Gy per fraction). Total doses in EBRT (to local lymph nodes and tumour bed) ranged from 50 to 60 Gy (1.8-2 Gy per fraction 5 days/week). One patient, treated with radical HDR brachytherapy, received 45 Gy in 10 fractions and $50 \mathrm{~Gy}$ in EBRT to regional lymph nodes.

Results: We did not notice local recurrences or distant metastases in our group of patients. Median follow-up was 67 months (range 47-79 months). All patients preserved normal tongue function. A severe late complication occurred in 1 patient - fracture of the mandible.

Conclusions: In the analysed group of young patients with mobile tongue cancer interstitial HDR brachytherapy in combination with EBRT was an effective and well tolerated treatment modality which allowed preservation of the tongue and its function.

J Contemp Brachyther 2010; 2, 2: $61-63$ DOI: 10.5114/jcb.2010.14403
\end{abstract}

Key words: tongue cancer, HDR brachytherapy, radiotherapy.

\section{Purpose}

Mobile tongue cancer is one of the most common squamous cell carcinomas located in the oral cavity [1, 2], affecting mostly older men with a history of long-term exposure to tobacco and alcohol [3]. Recently in many countries an increasing incidence of tongue cancer is observed, particularly among women and young men [3-7]. It is estimated that $3-6 \%$ of all tongue cancers appear in patients under 40 years $[4,6,8,9]$.

In early stage tongue cancer there is no standard treatment scheme and either surgery or radiotherapy can achieve a relatively similar overall survival rate [10]. Tumour resection is usually concerned with some postoperative cosmetics and functional tongue defects, i.e. in swallowing, chewing or speaking [6]. Definitive radio- therapy allows one to preserve tongue function, which is especially important among young patients. Radical radiotherapy in early stage tongue cancer can consist of EBRT (external beam radiotherapy) given to the tumour and/or local lymph nodes and brachytherapy. Sole interstitial brachytherapy or as a "boost" offers a high dose to the tumour while sparing normal tissues, short overall treatment time, good local control, preservation of tongue and good quality of life [11-14].

The role of LDR brachytherapy in mobile tongue cancer is well documented, but there is still only a limited number of papers devoted to HDR-BT $[7,15,16]$. In Gliwice Institute, in mobile tongue cancers, HDR-BT has been successfully used for many years either as a sole treatment or in combination with EBRT. 


\section{Material and methods}

In Maria Sklodowska-Curie Memorial Cancer Centre and Institute of Oncology, Gliwice Branch, from 2001 to 2006, 5 young patients (under 45 years) with early stage mobile tongue cancers were treated with HDR brachytherapy (T1NOM0 - 3/5 and T2NOM0 - 2/5). Medium age was 33.2 years (range $23-43$ yrs). There were $4 / 5$ women and $1 / 5$ man. Only one patient had been smoking, for 12 years. None abused alcohol. In histopathological examination in all cases squamous cell carcinoma was found (G1 and G2). One patient was treated previously with tumour resection, but because of positive surgical margins was referred for radiotherapy. All patients had clinically and in CT negative lymph nodes, without dissection. Interstitial brachytherapy was performed before EBRT. Catheters were inserted by a trocar needle into the tumour or tumour bed under general anaesthesia by a brachytherapist. The end of each catheter was switched to the surface of the tongue. During therapy dental care and shielding was used. HDR-BT was performed with an afterloading Ir192 source. The treatment planning was based on X-ray. The active lengths of catheters were marked on the radiographs and the catheter points were digitized into the PLATO-3D planning system. Reference points were set 5-8 $\mathrm{mm}$ from the catheters.

Patients were treated with interstitial HDR-BT (3-8 catheters). The number of catheters was determined by the extent of the target volume (tumour $+0.5-1 \mathrm{~cm}$ safety margin). Total doses in four patients treated with HDR-BT as a "boost" ranged from 18 to 21 Gy given in 6-7 fractions (twice a day, 3 Gy per fraction). EBRT was administered 2-3 weeks after brachytherapy. Total doses to local lymph nodes and tumour ranged from 50 to 60 Gy (1.8-2 Gy per fraction, 5 days/week). The spinal cord was shielded after $45 \mathrm{~Gy}$. No chemotherapy was administered in our group of patients. One patient who was treated with sole HDR brachytherapy received 45 Gy in 15 fractions (twice a day, 3 Gy per fraction) and then 50 Gy in EBRT to local lymph nodes ( 3 weeks after HDR-BT).

\section{Results}

During brachytherapy the following acute reactions were observed: temporary pain $(2 / 5)$, oedema $(1 / 5)$ and dysphagia (2/5). Most symptoms resolved within two weeks. Acute and late toxicity after EBRT was evaluated according to the Dische scale. The most common acute complications were: pain (3/5) and dysphagia (4/5). A severe late complication occurred in one patient - fracture of the mandible. Patients were followed up every 3-6 months after the end of therapy. The follow-up period from the beginning of treatment ranged from 47 to 79 months. There were no local failures or distant metastases in that group of young patients with mobile tongue cancer. All patients preserved normal tongue function.

\section{Discussion}

The main goal in early stage mobile tongue cancer is complete tumour removal and preservation of the organ with its function $[7,16,17]$. Because there is no standard treatment schedule, surgery, radiotherapy, chemoradiotherapy or combined treatment is possible $[6,14,18]$. The decision to choose a method should be made taking into account also the potential quality of life, especially in young patients with tongue cancer $[10,11,16]$. Primary surgery for early stage tongue cancers is usually concerned with tongue dysfunction and permanent disability [19]. Surgical ablation of tongue cancer may lead to severe impairment in speech, chewing or swallowing [20] and even recent progress in reconstructive surgery is usually not sufficient to restore the complex anatomy and function of the tongue [19]. Mobile tongue cancers can also be effectively treated with radiotherapy $[13,18]$. It was shown that in early stage tongue cancer both surgery with EBRT and EBRT with interstitial brachytherapy are effective at a similar level, with a local failure rate of $15-25 \%$ [6]. Interstitial brachytherapy is the most conformal technique which increases the dose to the target while sparing normal tissues $[7,11,12]$ and it is recommended by the American Brachytherapy Society as a component of the treatment in head-and-neck tumours [15]. The role and effectiveness of LDR brachytherapy in tongue cancer is very well documented, but there are only a limited number of papers on HDR-BT [7, 11, 13, 15].

In HDR-BT there is no standard treatment scheme, i.e. total doses, fractionation doses and treatment duration differ between centres $[1,7,11,13,21]$. In the literature there are reports of a high risk of complications [21] and bad local control in HDR-BT [22]. It is suggested that the role of HDR is controversial in tongue cancers because of the high rate of severe complications and poor local control $[7,11,22]$. In our twenty years of experience, including in tongue cancers, HDR-BT is a very good, effective and safe method of treatment [11]. We have our own scheme of treatment either in radical interstitial HDR-BT or as a "boost" with EBRT. Our fractionation dose in tongue cancer is 3 Gy twice a day up to a total dose of 45 Gy in radical HDR-BT and 18-21 Gy in combination with EBRT. It allows us to have small number of severe complications and good local control [11]. In our opinion the bad results in some studies with HDR-BT in early stage tongue cancers may be connected with too high dose per fraction and high total doses. It has been reported that about $80-90 \%$ of stage I and II tongue cancers could be locally cured with LDR-BT alone $[2,7,16]$. In our small group of young patients (under 45 years) no one developed local failure or lymph node metastases. In some publications there are also reports that HDR brachytherapy is an effective method with complication rates similar to low-dose-rate brachytherapy [12]. In some studies interstitial brachytherapy, especially HDR-BT, gives poor cosmetic and functional results in tongue preservation, causing hemiatrophy [22] and osteonecrosis [7, 13]. In our small group we observed fracture of the mandible only in one case.

Another very important issue is the neck lymph node status [14], because usually within a year they can develop metastases and in early cancer stages the rate of involved lymph nodes ranges between 8 and 33\% [10]. There is no standard treatment scheme in clinically nega- 
tive neck lymph nodes. Either elective radiotherapy or surgical dissection is possible, but in some hospitals a watching policy is preferred $[14,17,18]$. In our department neck lymph nodes are irradiated, usually up to $50 \mathrm{~Gy}$. Patients in our small group did not develop lymph node metastases.

In the literature there are suggestions that oral cavity cancers can be more aggressive among young patients [ 4 , $6,8,9]$ and that this group may need special procedures. Recently it was found that in contrast to the older population, young people reported never having smoked or consumed alcohol excessively [3]. It is suggested that there are possible other risk factors for carcinogenesis, i.e. exposure to formaldehyde, chronic immune defects, immunosuppression or some viral infections such as HIV, HPV and EBV $[3,4,23]$.

We may expect that the number of young patients with tongue cancers who require good and effective therapy with organ preservation strategies will increase. In our opinion interstitial HDR-BT in carefully selected patients with individual treatment planning may be a very good option.

\section{Conclusions}

In our group of young patients with mobile tongue cancer interstitial high-dose-rate brachytherapy in combination with EBRT was an effective and well tolerated treatment modality which allowed preservation of the tongue and its function.

\section{References}

1. Chen AY, Myers JN. Cancer of the oral cavity. Dis Mon 2001; 47: 275-361.

2. Bagan J, Sarrion G, Jimenez Y. Oral cancer: clinical features. Oral Oncol 2010; 46: 414-417.

3. Llewellyn CD, Johnson NW, Warnakulasuriya KA. Risk factors for squamous cell carcinoma of the oral cavity in young people - a comprehensive literature review. Oral Oncol 2001; 37: 401-418.

4. Llewellyn CD, Linklater K, Bell J et al. An analysis of risk factors for oral cancer in young people: a case-control study. Oral Oncol 2004; 40: 304-313.

5. Bunnell A, Pettit N, Reddout N et al. Analysis of primary risk factors for oral cancer from select US states with increasing rates. Tob Induc Dis 2010; 8: 5 .

6. Liao CT, Wang HM, Hsieh LL et al. Higher distant failure in young age tongue cancer patients. Oral Oncol 2006; 42: 718-725.

7. Umeda M, Komatsubara H, Ojima Y et al. A comparison of brachytherapy and surgery for the treatment of stage I-II squamous cell carcinoma of the tongue. Int J Oral Maxillofac Surg 2005; 34: 739-744.

8. Warnakulasuriya S, Mak V, Möller H. Oral cancer survival in young people in South East England. Oral Oncol 2007; 43: 982-986.

9. Ribeiro AC, Silva AR, Simonato LE et al. Clinical and histopathological analysis of oral squamous cell carcinoma in young people: A descriptive study in Brazilians. $\mathrm{Br} \mathrm{J}$ Oral Maxillofac Surg 2009; 47: 95-98.

10. Ichimiya $Y$, Fuwa N, Kamata $\mathrm{M}$ et al. Treatment results of stage I oral tongue cancer with definitive radiotherapy. Oral Oncol 2005; 41: 520-525.
11. Sasiadek W, Bialas B, Fijalkowski M et al. Interstitial HDRbrachytherapy in patients with oral cavity cancers - preliminary treatment results based upon material of Institute of Oncology in Gliwice. Nowotwory 2007; 57: 542-546 [in Polish].

12. Guinot JJ, Santos M, Tortajada MI et al. Efficacy of high-doserate interstitial brachytherapy in patients with oral tongue carcinoma. Brachytherapy 2010 [article in press]; DOI: 10.1016/ j.brachy.2009.10.003.

13. Umeda M, Komatsubara H, Nishimatsu N et al. High-dose rate interstitial brachytherapy for stage I-II tongue cancer. Oral Surg Oral Med Oral Pathol Oral Radiol Endod 2000; 90: 667-670.

14. Bourgier $C$, Coche-Déquéant B, Fournier $C h$ et al. Exclusive low-dose-rate brachytherapy in 279 patients with T2N0 mobile tongue carcinoma. Int J Radiat Oncol Biol Phys 2005; 63: $434-440$.

15. Nag S, Cano ER, Demanes DJ et al. The American Brachytherapy Society recommendations for high-dose-rate brachytherapy for head-and-neck carcinoma. Int J Radiat Oncol Biol Phys 2001; 50: 1190-1198.

16. Yoshimura R, Shibuya H, Miura M et al. Quality of life of oral cancer patients after low-dose-rate interstitial brachytherapy. Int J Radiat Oncol Biol Phys 2009; 73: 772-778.

17. Nakagawa T, Shibuya H, Yoshimura R et al. Neck node metastasis after successful brachytherapy for early stage tongue carcinoma. Radiother Oncol 2003; 68: 129-135.

18. Watanabe H, Mogushi K, Miura M et al. Prediction of lymphatic metastasis based on gene expression profile analysis after brachytherapy for early-stage oral tongue carcinoma. Radiother Oncol 2008; 87: 237-242.

19. Matsui K, Ohno Y, Yamashita K et al. Factors influencing postoperative speech function of tongue cancer patients following reconstruction with fasciocutaneous/myocutaneous flaps a multicenter study. Int J Oral Maxillofac Surg 2007; 36: 601-609.

20. Matsui $Y$, Shirota T, Yamashita $Y$ et al. Analyses of speech intelligibility in patients after glossectomy and reconstruction with fasciocutaneous/myocutaneous flaps. Int J Oral Maxillofac Surg 2009; 38: 339-345.

21. Lau HY, Hay JH, Flores AD et al. Seven fractions of twice daily high dose-rate brachytherapy for node-negative carcinoma of the mobile tongue results in loss of therapeutic ratio. Radiother Oncol 1996; 39: 15-18.

22. Yoshioka Y, Yoshida K, Shimizutani K et al. Proposal of a new grading system for evaluation of tongue hemiatrophy as a late effect of brachytherapy for oral tongue cancer. Radiother Oncol 2001; 61: 87-92

23. Yen CY, Lu MC, Tzeng CC et al. Detection of EBV infection and gene expression in oral cancer from patients in Taiwan by microarray analysis. J Biomed Biotechnol 2009; 2009: 904589. 